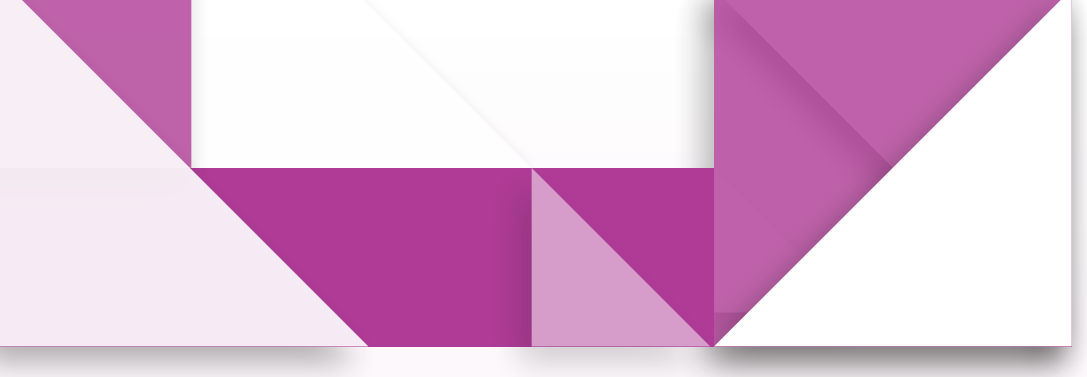

\title{
Enseñanza de la cinética 1 química por medio de simulaciones y aprendizaje activo
}

- Teaching Chemical Kinetics through Simulation and Active Learning

- Ensino da cinética química por médio de simulações e aprendizagem ativa
Julián Guillermo Paredes-Navia* Manuel Fredy Molina-Caballero**

\begin{abstract}
Resumen
Este artículo de investigación presenta una propuesta de implementación de actividades experimentales y de simulación, utilizando como fundamento los principios de la metodología del aprendizaje activo, para la enseñanza-aprendizaje de los factores que modifican la velocidad de una reacción química. La estrategia se aplica en los grados décimos de la Institución Educativa San Lorenzo (IESL) del municipio de Suaza en el departamento del Huila (Colombia). Para ello se toman dos grupos, uno de los cuales integra el desarrollo de las actividades a través del trabajo práctico y, otro, que lo hace utilizando el simulador de experimentos de cinética química "virtual LabCin". La propuesta integra la aplicación de un test de ideas previas, el desarrollo de talleres de interacción-demostración y la implementación de la prueba final. Conforme a los resultados, se hace un análisis de confrontación temporal (antes, durante y después), en el cual se determina que la propuesta didáctica no solo permite establecer un impacto positivo y favorable en el rendimiento y adquisición de conocimiento de los conceptos abordados, sino que también proporciona el desarrollo de capacidades intelectuales y procedimentales, que estimulan la proposición, la confrontación, la comunicación y el desarrollo de ideas.
\end{abstract}

Palabras clave

experimentación; simulación; aprendizaje activo; cinética química.
Magíster en Enseñanza de las Ciencias Exactas y Naturales, Universidad Nacional de Colombia, sede Bogotá. Docente de Química, Física y Biología, Secretaría de Educación del Huila, Colombia. igparedesn@unal.edu.co

Orcid.org/0000-0003-0169-3681

** Magíster en Ciencias y Química, docente asociado de la Facultad de Ciencias, Departamento de Química, Universidad Nacional de Colombia, sede Bogotá, Colombia.

mfmolinac@unal.edu.co

Orcid.org/0000-0001-8758-2637 


\begin{abstract}
This research paper presents a proposal for the implementation of experimental and simulation activities, based on the principles of active learning methodology, for teaching and learning the factors that modify the rate of reaction. This strategy was applied to tenth graders from San Lorenzo Secondary School, located in the town of Suaza, in Huila department (Colombia). For this purpose, two groups are taken, one of which integrates the development of the activities through the practical work and another one, which does so using the "Virtual LabCin" chemical kinetic experiments simulator. The proposal integrates the application of a previous ideas test, the development of interaction-demonstration workshops, and the implementation of the final test. According to the results, a temporal confrontation analysis (before, during and after) is run. This analysis determines that the didactic proposed not only allows establishing a positive and favorable impact on the performance and acquisition of knowledge of the concepts addressed, but also provides the development of intellectual and procedural capacities, which stimulate proposition, confrontation, communication and the development of ideas.
\end{abstract}

Keywords

\title{
Resumo
}

Este artigo de pesquisa apresenta uma proposta para a implementação de atividades experimentais e de simulação, baseadas nos princípios da metodologia de aprendizagem ativa, para o ensino-aprendizagem dos fatores que modificam a velocidade de uma reação química. A estratégia é aplicada na décima série da Instituição Educacional San Lorenzo do município de Suaza, no departamento de Huila (Colômbia). Para isso, dois grupos são conformados, um dos quais integra o desenvolvimento de atividades através de trabalhos práticos e outro, que utiliza o simulador de experimentos de cinética química "virtual LabCin". A proposta integra a aplicação de um teste de ideias prévias, o desenvolvimento de oficinas de interação-demonstração e a implementação do teste final. De acordo com os resultados, realizamos uma análise de confronto temporal (antes, durante e depois), em que determinamos que a abordagem didática não só permite estabelecer um impacto positivo e favorável sobre o desempenho e a aquisição de conhecimento dos conceitos abordados, mas também fornece o desenvolvimento de capacidades intelectuais e processuais, que estimulam a proposição, o confronto, a comunicação e o desenvolvimento de ideias.

Palavras-chave

experimentação; simulação; aprendizagem ativa; cinética química 


\section{Introducción}

En el proceso de enseñanza-aprendizaje de la química, el desarrollo de la experimentación y del trabajo práctico asociado a la incorporación de herramientas tecnológicas se convierten en una estrategia que permite mejorar el ritmo de aprendizaje de los estudiantes (Kaya y Geban, 2012), la gestación de competencias que tienen que ver con el uso de los conocimientos de química, su aplicación en contexto y la utilización de las nuevas tecnologías. Sin embargo, la dificultad para poder comprender estos principios radica en gran medida en que la aplicación práctica que se le da a estos es, por lo general, nula.

No obstante, la realidad es que el desarrollo de estas habilidades no se está implementando transversalmente en la enseñanza, especialmente en temas en que es importante su utilización, como es el caso de la cinética química. Este contenido se aborda en el grado décimo, en el que se han encontrado dificultades para su comprensión, debido al uso de metodologías tradicionales que no satisfacen las necesidades de aprendizaje de los estudiantes (Tejada, Chicangana y Villabona, 2013) y excluyen la experimentación, la utilización de las TIC y la modelización científica como aspectos fundamentales en la enseñanza de la química (Cardellini, 2012; Kurt y Ayas, 2012; Lynch, 1997).

La presencia e importancia del trabajo práctico, el uso de las TIC y la construcción de modelos científicos en el aula, fundamentados en una metodología de aprendizaje activo, se constituyen en una buena opción para responder de la mejor forma posible a esta realidad, de tal manera que permita generar la capacidad de organizar y promover las ideas que expresan los estudiantes de su mundo cotidiano (Acher, 2014, pp. 67-68). En efecto, esta pedagogía del aprendizaje activo pretende facilitarle al docente el uso de herramientas para motivar el aprendizaje de los alumnos, integrando una secuencia de actividades que involucra la formulación de predicciones, la observación directa, la realización de actividades prácticas, la discusión y síntesis de las ideas o conceptos comprendidos de los fenómenos naturales, en este caso, relacionados con la cinética química. De esta manera, se busca finalmente tratar de cambiar en los alumnos las representaciones mentales erróneas que suelen tener de los conceptos de la incidencia de los factores en la velocidad de las reacciones químicas, y que sean ellos mismos quienes logren vincular lo que ya saben con los elementos nuevos, utilizando su propio esfuerzo y parte de los materiales de los que ya disponen (Ramos, 2013).

En consecuencia, esta propuesta surge con el objetivo de implementar actividades que relacionen el trabajo práctico con la teoría en cinética química para proporcionar experiencias directas sobre fenómenos (Barberá y Valdés, 1996), se permita el cambio de las concepciones de los estudiantes a partir de la confrontación entre sus observaciones y sus creencias y se haga uso de la tecnología a través de la utilización de herramientas virtuales y experimentales buscando ambientes de aprendizaje acordes al conocimiento científico, en el que se genere en los alumnos la posibilidad de interactuar con otros, fortalecer el trabajo en equipo y facilitar una participación activa.

\section{Antecedentes}

\section{Investigación didáctica de la cinética química}

Los estudios en didáctica de la química, han demostrado que los conceptos de cinética química son considerados temas altamente relevantes para la enseñanza de las reacciones 
químicas y del equilibrio químico (Kaya y Geban, 2012, p. 217; Kurt y Ayas, 2012, p. 980; Sánchez, Domínguez y García, 2002, p. 172), además, revelan que debe ser estructurado como contenido central en el plan de estudios de química (Cachapuz y Maskill, 1987, tomado de Kaya y Geban, 2012, p. 217; MEN, 2004, p. 22), pues permite que los estudiantes aprendan cómo se produce una reacción química por medio de la teoría de las colisiones, la importancia de los catalizadores, los mecanismos de las reacciones y sus aplicaciones industriales (Tastan, Yalçinkaya y Boz, 2010, p. 47). No obstante, se ha encontrado que no hay muchas investigaciones acerca de la comprensión de los conceptos de velocidad de reacción, ya que la mayoría de los estudios en enseñanza de la cinética química está bajo la sombra del equilibrio químico (Sánchez, Domínguez y García, 2002, p. 172).

Así mismo, las investigaciones internacionales ponen de manifiesto que los estudiantes de los diferentes niveles académicos, tienen problemas en la explicación de los conceptos científicos que se observan en la vida cotidiana (Wu, 2003) y el concepto de velocidad de reacción es considerado uno de los temas con el que los estudiantes tienen dificultades para entender y aplicar a la vida real (Kurt y Ayas, 2012, p. 980).

Dentro de las propuestas que se han utilizado para mejorar la enseñanza y aprendizaje de la cinética química se encuentran en su mayoría estudios con metodologías que permitieron dar a conocer los diferentes errores conceptuales que tienen los estudiantes y profesores de la temática de la velocidad de las reacciones químicas y sobre su posible origen (Ahiakwo e Isiguzo, 2015; Kolomuç y Çalik, 2012; Kolomuç y Tekin, 2011 ; Tastan, Yalçinkaya y Boz, 2010), a su vez se han utilizado estrategias basadas en el modelo constructivista con actividades de resolución de problemas de los conceptos de cinética química y su aplicación en acontecimientos de la vida real (Kurt y Ayas, 2012); el uso de actividades y trabajos prácticos o experimentales para estudiantes y profesores siguiendo el enfoque de la enseñanza y aprendizaje significativo (Barrera, 2013, p.175; Kaya y Geban, 2012; Martínez, 2009); la utilización de modelos y analogías como estrategia para aproximar lo abstracto mediante representaciones concretas (Bender, Cutrera y Defago, 2007) y el uso de recursos informáticos que van desde la utilización de simuladores que modelan los procesos ocurridos en las reacciones micro (Bigger, 2011 ; Da Silva, Sousa, Silva, Oliveira y Melo, 2014; Huddle y White, 2000; Stieff y Wilensky, 2003), el manejo de hojas de cálculo de la plataforma Ms Excel conveniente para conceptualizar datos cinéticos experimentales: trazar gráficos de forma rápida, determinar el orden de una reacción y la constante de velocidad (Zain, Abdul y Sui, 2013), el diseño de objetos virtuales de aprendizaje (OVA) como herramientas para complementar las actividades planeadas por el docente dentro de su estrategia de enseñanza al abordar el tema de cinética química (Botero y Palomeque, 2014) y la implementación de las TIC (Hernández, 2013). 


\section{Marco teórico}

\section{Metodología de aprendizaje activo}

El aprendizaje activo se define como un método de enseñanza en donde el eje central del proceso de aprendizaje son los estudiantes, a través de la realización de actividades prácticas en el aula, que permiten que los alumnos piensen en lo que están haciendo (Bonwell y Eison, 1991, citado de Prince, 2004, p. 1). Los elementos centrales del aprendizaje activo son las actividades de los estudiantes y su compromiso en el proceso de aprendizaje (Prince, 2004, p. 1).

A diferencia de la clase tradicional en donde los estudiantes reciben pasivamente la información por parte del profesor, el aprendizaje activo conduce a mejorar las actitudes de los alumnos, en el desarrollo de habilidades de pensamiento crítico. Según Hiler y Paul (2006), para aprender se debe pensar y preguntar continuamente: “¿Qué quiere decir esto realmente? ¿Cómo lo sabemos? Si es cierto, ¿̇qué más es cierto?" y contestar estas preguntas por sí mismo. Las respuestas que el profesor da no se asimilan completamente a menos que las mentes de los estudiantes estén listas para recibirlas. El fundamento de la estrategia radica en propiciar una actitud activa del estudiante durante la dinámica de la clase, en donde él mismo es autónomo, responsable y consciente de lo que aprende, de qué debe aprender y de lo que no ha aprendido (Sierra, 2013).

Este método exige que los educandos no sean simples espectadores, que no se limiten simplemente a oír, sino que generen procesos de lectura, escritura, indagación y resolución de problemas. Es decir, según Gonzales (2000), le permite la utilización de operaciones intelectuales de orden superior como el análisis, la síntesis, la interpretación, la inferencia y la evaluación. En consecuencia, se requiere por parte del profesor una forma diferente de conducir la clase, y dejar atrás la cátedra expositiva, en la que se caracteriza por ser el eje central del proceso de enseñanza-aprendizaje. Por tanto, el docente adquiere el rol de facilitador, orientando a los alumnos en su proceso de búsqueda del conocimiento (Cuadrado, 2010, p. 2).

\section{La experimentación en el aula de clase}

Uno de los enfoques utilizados con mayor impacto en la enseñanza-aprendizaje de las ciencias naturales, entre ellos la química, especialmente en temáticas donde prevalece la práctica, es considerar la indispensable relación entre teoría y experimentación. De esta manera, se puede razonar que el aula debe convertirse en un espacio de generación de conocimiento, en donde prevalezca la indagación y la experimentación constante y se aleje un poco del uso memorístico y repetitivo de fórmulas. Sin embargo, según Golombek (2008), es estrictamente cierto que el enfoque experimental de la enseñanza de las ciencias naturales requiere una base metodológica que permita la formulación de tales experimentos, de forma que el docente pueda encaminar al estudiante por un proceso de descubrimiento.

Por consiguiente, los trabajos prácticos o experimentales son actividades diseñadas para dar a los estudiantes la oportunidad de acercarse al trabajo que realizan los científicos en la resolución de problemas, que pueden ser investigaciones teóricas y/o prácticas (Albadalejo y Caamaño, 1992, pp. 95-157), en el que los estudiantes siguen un procedimiento con el fin de demostrar, corroborar y convencerse de una teoría o ley científica. 


\section{Simuladores y laboratorios virtuales en la enseñanza de las ciencias naturales}

El uso de las TIC en la enseñanza abre un abanico de nuevas posibilidades en la utilización de herramientas informáticas que rompen con esquemas tradicionales y revelan nuevas vías pedagógicas sustentadas en la participación activa de los estudiantes, lo que enriquece de manera notable el proceso de enseñanza-aprendizaje (Useda y Castañeda, 2015, p. 126). Uno de estos materiales que hace parte de los cambios históricos impuesto por la evolución tecnológica, que ha influido significativamente no solo en educación sino también en otros sectores como la medicina, la economía, la ingeniería, entre otras, es el uso y la implementación de los simuladores.

Los simuladores utilizados en educación se definen como "programas que tienen un modelo de algún aspecto del mundo y que permite al estudiante cambiar algunos parámetros o variables de entrada, ejecutar o correr el modelo y desplegar los resultados" (Escamilla, 2000, citado de Contreras y Carreño, 2012, p. 108). Para Narváez (2015, p. 29), "los simuladores son herramientas que llevan a los estudiantes a imitar un contexto real, estableciendo en ese ambiente situaciones problemáticas o reproductivas, similares a las que él deberá enfrentar".

Su uso en la enseñanza de las ciencias naturales, en especial, de la química, permite además de un cambio de ambiente, mejorar y complementar la enseñanza y aprendizaje de los conceptos químicos en los que los estudiantes han presentado problemas para comprender. Debido a que los simuladores permiten la modelación de prácticas científicas, han logrado una gran adaptabilidad a los espacios destinados a la experimentación en el ámbito educativo, dando lugar a laboratorios virtuales de aprendizaje. Según Cataldi, Donnamaría y Lage (2008, p. 7), este medio tecnológico facilita la tarea de involucrar a estudiantes y profesores en el método científico, convirtiendo al trabajo de laboratorio y sus precauciones por accidentes, en una opción de aprendizaje en donde el alumno puede equivocarse y repetir la actividad con una inversión baja. Para Rodríguez, Mayén, Rodríguez y Ruiz (2013), los laboratorios virtuales también se pueden utilizar como medio de preparación para adquirir o fortalecer las habilidades, destrezas y conocimientos básicos, seguir con el buen uso de las clases prácticas y evaluar las habilidades que se deben de tener en un laboratorio real.

\section{Metodología}

El estudio es de tipo descriptivo-comparativo, que busca, según Dunkhe (1986), especificar las propiedades importantes de personas, grupos, comunidades, 0 cualquier otro fenómeno que sea sometidos a un análisis (tomado de, Fernández y Baptista, 2003, p. 45), y esto es lo que se efectuó en este estudio, se determinó la incidencia de la práctica experimental y la simulación en el aprendizaje de los estudiantes, del tema: factores que afectan la velocidad de una reacción química, 
a través de la aplicación de un tratamiento (estrategia de enseñanza-aprendizaje), lo que permitió evaluar el fenómeno y describirlo lo más ampliamente posible.

La investigación se ajusta a un diseño cuasi experimental porque se trata de un estudio pre/postest y por la forma no aleatoria de seleccionar a las dos poblaciones de estudio, los cuales ya estaban formadas antes de la experiencia. El propósito consiste en observar el efecto que tiene la aplicación del tratamiento de la estrategia didáctica en sus versiones respectivas para las dos secciones, sobre el aprendizaje de los conceptos propuestos, es decir que no hay una designación de tipo experimental y control.

\section{Contexto educativo y población}

La estrategia didáctica se aplica en la Institución Educativa San Lorenzo ubicada en el municipio de Suaza. El colegio hace parte del organismo pedagógico oficial de la Secretaría de Educación del departamento del Huila. La población de este estudio está compuesta por estudiantes de los grados décimos, en los que la mayoría de jóvenes se establecen en el primer y segundo estrato socioeconómico. Para su ejecución se tienen en cuenta dos grupos que son la base para analizar el efecto de la propuesta. En el primero, con censo estudiantil de 36 estudiantes del grado 10A, se realizan clases con la aplicación de trabajos experimentales, este se designa como GTE. En el segundo, con una población estudiantil de 26 jóvenes del grado 10B, se ejecuta una propuesta basada en el uso de prácticas de simulación a través del software "virtual LabCin", esta sección es denominada gps. En ambos grupos se trabaja el mismo tema, utilizando una metodología basada en los criterios del aprendizaje activo.

\section{Desarrollo de la investigación}

El marco metodológico se fundamenta en cuatro fases principales (tabla 1), durante las cuales se proponen los instrumentos, se desarrollan las actividades y se recolecta la información necesaria para el análisis y el cumplimiento de los objetivos descritos.

Tabla 1. Etapas del estudio

\begin{tabular}{|c|c|c|}
\hline N. ${ }^{\circ}$ & $\begin{array}{l}\text { Fases de la } \\
\text { investigación }\end{array}$ & Acciones \\
\hline \multirow{6}{*}{1} & \multirow{6}{*}{ Planificación } & Documentación bibliográfica \\
\hline & & Realización del test \\
\hline & & $\begin{array}{l}\text { Diseño y desarrollo del simulador } \\
\text { Virtual LabCin }\end{array}$ \\
\hline & & $\begin{array}{l}\text { Realización de manuales de } \\
\text { práctica y simulación }\end{array}$ \\
\hline & & Validación de los instrumentos \\
\hline & & $\begin{array}{l}\text { Utilización de escenarios y } \\
\text { plataformas tecnológicas de } \\
\text { enseñanza-aprendizaje }\end{array}$ \\
\hline \multirow{3}{*}{$2 / 3$} & \multirow{3}{*}{$\begin{array}{l}\text { Ejecución/ } \\
\text { observación }\end{array}$} & $\begin{array}{l}\text { Reconocimiento de ideas previas } \\
\text { e introducción (aplicación del } \\
\text { pretest) }\end{array}$ \\
\hline & & $\begin{array}{l}\text { Desarrollo de actividades } \\
\text { experimentales y uso del } \\
\text { simulador }\end{array}$ \\
\hline & & $\begin{array}{l}\text { Prueba final (aplicación del } \\
\text { postest) }\end{array}$ \\
\hline 4 & Reflexión & $\begin{array}{l}\text { Análisis y conclusiones de la } \\
\text { propuesta }\end{array}$ \\
\hline
\end{tabular}

Fuente: elaboración propia.

\section{Fase de planificación}

- Documentación bibliográfica: esta primera actividad consiste en una considerable revisión bibliográfica, a fin de originar los diferentes marcos que componen este trabajo: el marco teórico disciplinar, epistemológico y el marco metodológico que provee los diferentes procedimientos investigativos. Esta etapa es constante en todo el proceso y se actualiza durante la última fase. 
- Realización del test: con el propósito de determinar el conocimiento que los alumnos tenían acerca de los conceptos seleccionados antes y después de aplicar la estrategia didáctica, se construye un test con 14 preguntas de selección múltiple con única respuesta. Para su realización se tiene como referencia el trabajo de investigación de Cakmakci y Aydogdu (2011), el cuestionario de Huamán (2014), el banco de preguntas de química del Icfes y conocimientos propios del tema.

- Diseño y desarrollo del simulador Virtual LabCin: este laboratorio virtual de cinética química es un software de creación propia desarrollado en la plataforma Unity3D, que funciona principalmente como motor de videojuegos, sin embargo, su completo contenido de herramientas de animación en 2D y 3D también permite la creación de simuladores. Su programación se fundamenta en la implementación del código .NET Framework a través del lenguaje C\#.

El simulador cuenta con una interfaz que le permite al usuario disponer de materiales, instrumentos, reactivos y de los procedimientos — basados en la metodología del aprendizaje activo- que se deben de tener en cuenta para la ejecución de los experimentos. Por el momento, el programa se puede utilizar en ordenadores y tabletas con el sistema operativo Windows.

- Realización de manuales de práctica y simulación: teniendo como base la metodología del aprendizaje activo, se construyen cinco guías para las actividades de experimentación y uso del laboratorio virtual, nombradas como manuales de prácticas y manuales de simulación.

Los manuales de práctica y simulación están establecidos por cinco experimentos, en donde se permite identificar los conceptos de los factores que modifican la velocidad de una reacción química.

- Validación de los instrumentos: para garantizar que los instrumentos utilizados respondan a los objetivos de la investigación, establecer que estos sean considerados idóneos y se puedan utilizar con toda confianza, se hace necesario su validación. Esta determina el grado de confiabilidad y validez de los instrumentos (González, 2008, citado por Bojórquez, López, Hernández y Jiménez, 2013, p. 2).

La confiabilidad se comprueba a través del método estadístico alfa de Cronbach, que es el promedio de las correlaciones entre los ítems que hacen parte de un instrumento (Streiner, 2003, citado por Oviedo y Campo, 2005) y la validez se realiza a través de la valoración de investigadores y expertos, en este caso, docentes y profesionales del campo de la química. Esta evaluación se analiza a través del índice de Bellack y el coeficiente de validez $\mathrm{V}$ de Aiken, que permiten cuantificar el acuerdo entre jueces.

- Utilización de escenarios y plataformas tecnológicas de enseñanza y aprendizaje: para propiciar el adecuado desarrollo de las actividades de experimentación 
y simulación, se dispuso de algunos espacios físicos y virtuales, que permitieron establecer las condiciones apropiadas para la enseñanza y aprendizaje de los conceptos seleccionados.

En los escenarios físicos se utilizaron las aulas o salones de las dos poblaciones de estudio, el laboratorio de química y la sala de bilingüismo del colegio, que dispone de computadoras. Por otra parte, el ambiente virtual se creó mediante el uso de las tecnologías de la información y la comunicación (TIC), en donde se usan computadoras, tabletas, video beam y se emplea el software informático para la enseñanza de conceptos de cinética química, Virtual LabCin. Igualmente, se utiliza la plataforma virtual Génesis VLE, la cual permite administrar y compartir toda clase de archivos a las computadoras de los estudiantes desde un solo ordenador, de tal forma que toda la información queda al alcance de ellos.

\section{Fases de ejecución y observación}

Reconocimiento de ideas previas e introducción (aplicación del pretest): esta actividad inicia con un ejercicio de diagnóstico, empleando como instrumento de indagación de ideas previas, la aplicación del pretest. Este se aplica de forma física para el grupo de trabajo experimental (GTE), y de forma virtual para el grupo de prácticas de simulación (GPS), a través de la plataforma Génesis VLE. Los resultados del pretest son tabulados, analizados y evaluados para determinar el conocimiento del alumno sobre los conceptos de los factores que afectan la velocidad de las reacciones. También, la prueba de diagnóstico tiene el objetivo de comparar el nivel de los estudiantes de ambos grupos antes de aplicar las actividades de la estrategia, para medir si son iguales o hay diferencias entre ellos que puedan incidir en los resultados.

Desarrollo de actividades experimentales y uso del simulador Virtual LabCin: la dinámica de las clases, tanto para el grupo de trabajo experimental como para el grupo en donde se utilizó el simulador, está guiada respectivamente por los cinco manuales de prácticas y de simulación, en donde el docente y los estudiantes siguen cada uno de los pasos (tabla 2) que caracterizan a las clases teóricas demostrativas y las actividades interactivas del aprendizaje activo (adaptado de Sokoloff, 2006, p. 5). Esta guía de acción es un complemento a la metodología establecida en el proyecto "Active Learning in Optics and Photonics-ALOP" patrocinado por la Unesco (Alarcón et al., s. f.).

Tabla 2. Pasos para las clases teóricas demostrativas y actividades interactivas del aprendizaje activo

a. Introducción y contextualización: pretende ubicar y situar a los estudiantes en un contexto determinado. El docente describe el experimento y lo explica sin proyectar el resultado.

b. Formulación de predicciones: los estudiantes deben registrar su predicción individual, es decir, lo que considera que va a suceder al ejecutar la situación problema. El docente debe enfatizar que sus respuestas no se tendrán en cuenta para la evaluación. Luego los estudiantes discuten sus predicciones en un grupo de tres compañeros, se designa a un relator, quien registrará y expresará la predicción final del grupo. Por último, el docente recoge verbalmente o por escrito las predicciones de cada grupo.

c. Actividad y observación: los alumnos y/o el profesor realizan la práctica mostrando claramente los resultados.

d. Discusión: se pide a algunos estudiantes que describan los resultados y se discutan en el contexto de la demostración para luego ser registrados como resultados.

e. Síntesis: los estudiantes o el docente realizan una síntesis de los conceptos involucrados en los resultados analizados, para este se discuten situaciones análogas con características que respondan al mismo concepto.

Fuente: elaboración propia. 
Aplicación de la prueba final (postest): finalmente, se evalúa el desempeño de los dos grupos y el conocimiento adquirido en el desarrollo de la estrategia didáctica por medio de un postest, que consta de las mismas preguntas establecidas en la primera prueba, con el objetivo de valorar todo un proceso.

\section{Fase de reflexión}

Con el propósito de dar respuestas puntuales a la problemática de enseñanza y aprendizaje de los conceptos de cinética química, se hace necesario establecer una fase de reflexión que permita evaluar los resultados establecidos antes, durante y después del desarrollo de la estrategia didáctica. Es importante reconocer también que una buena forma de valorar la práctica del docente, en este caso, en la enseñanza de los contenidos establecidos, es a través de la evaluación del desempeño de los estudiantes y de los resultados que arrojaron las pruebas presentadas. Esta última fase incluye el análisis general de la propuesta y las conclusiones.

\section{Plan de análisis}

El análisis de los datos se hace en su gran mayoría de manera descriptiva, utilizando la comparación de los resultados del pre y postest que presentan los estudiantes de los dos grupos. Para ello se emplean tres métodos principales. El primero se basa en utilizar la interpretación de gráficas porcentuales de los resultados del pre y postest de cada ítem de las pruebas para los dos grupos. También se establece una escala de valoración que permite estimar el desempeño y la competencia obtenida por los estudiantes en el pre/postest teniendo en cuenta el puntaje obtenido en el rango de 0 a 5 . De esta manera, se realiza la comparación de los grupos de acuerdo con el nivel de logro alcanzado. Finalmente, se hace uso del método estadístico de diagramas de cajas y bigotes para analizar la simetría, dispersión, la tendencia central de los valores obtenidos en las pruebas de los dos grupos, y además poder comparar los resultados del pretest con los de la prueba final.

\section{Resultados y análisis}

\section{Fase de planificación}

La fase de planificación incluye las actividades de diseño y elaboración de los instrumentos para el desarrollo de la estrategia didáctica y los aspectos relacionados con la etapa de validación.

La validación se hace de los tres instrumentos (test de ideas previas, los cinco manuales de prácticas-simulación y el software o laboratorio virtual) que fueron diseñados para la recolección de datos y el cumplimiento de los objetivos de la investigación. 
Para el cuestionario se realiza la aplicación de la prueba piloto en el grado décimo " $\mathrm{C}$ " teniendo en cuenta que este grupo tiene características similares a las de las poblaciones de estudio. Se realiza el análisis estadístico a través de alfa de Cronbach de las apreciaciones hechas por los estudiantes para cada pregunta, al igual que la evaluación del test por expertos por medio del índice de Bellack y del coeficiente de validez $\mathrm{V}$ de Aiken, indicando que este instrumento es confiable y válido, y además permite la consecución de los objetivos de la investigación. Sin embargo, el cuestionario es sometido a una reestructuración atendiendo a las sugerencias y recomendaciones de los estudiantes y jueces que evaluaron este instrumento.

Por su parte, los talleres utilizados para realizar las clases teóricas demostrativas y las actividades interactivas del aprendizaje activo de forma práctica y simulada fueron evaluados por jueces expertos (docentes de química). Este juicio manifiesta algunas observaciones referentes a la redacción y orden de acuerdo con el proceso que identifica el aprendizaje activo, no obstante, se determina la aprobación y aplicación del instrumento, a través de los métodos estadísticos de validación.

El laboratorio virtual fue sometido al juicio de cuatro expertos (docentes de ciencias, ingenieros y químicos), seleccionados por su conocimiento en la temática y de las características del instrumento. En total se hicieron catorce modificaciones al lenguaje de programación del laboratorio virtual atendiendo a las observaciones de los jueces y a su previa ejecución. Por consiguiente, se tomaron todas las medidas pertinentes para la reestructuración del programa, con el objetivo de brindarle confiabilidad y validez al instrumento.

\section{Fases de ejecución y observación}

Estas dos fases incluyen las actividades de reconocimiento de ideas previas, introducción a la temática, el desarrollo de los talleres de experimentación y simulación, y la aplicación de la prueba final.

Análisis del pretest considerando el nivel de desempeño

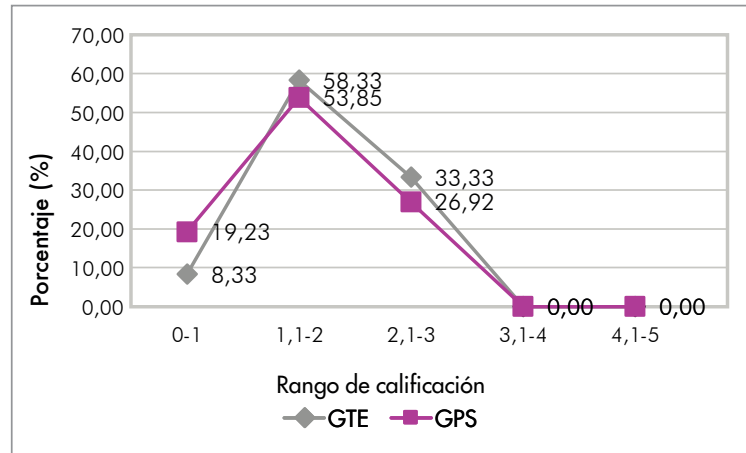

Figura 1. Relación entre el porcentaje y las calificaciones obtenidas por los dos grupos en el pretest Fuente: elaboración propia.

La figura 1 muestra los resultados del test de ideas previas teniendo en cuenta el rango de calificación, la distribución de estudiantes en cada uno de estos rangos y el porcentaje establecido para los dos grupos. Según la gráfica, en el GTE el nivel de logro alcanzado fue un poco mayor que el GPS, con una diferencia considerable en el rango $1,1-2$ y $2,1-3$, de $4,49 \%$ y $6,41 \%$ respectivamente. Por tanto, los conocimientos previos de la mayoría de estudiantes de los dos grupos están en la escala no satisfactorio, es decir que la comprensión de los conceptos acerca de los factores que afectan la velocidad de las reacciones químicas y su aplicación en la resolución de problemas es escasa. Por otra parte, se muestra un porcentaje significativo en la escala aceptable, reportando un valor del 33,33\% en el GTE y del 26,92\% en el GPS, asumiendo que estos estudiantes presentan dificultades para relacionar los conceptos en situaciones problémicas. 


\section{Análisis del postest considerando el nivel de desempeño}

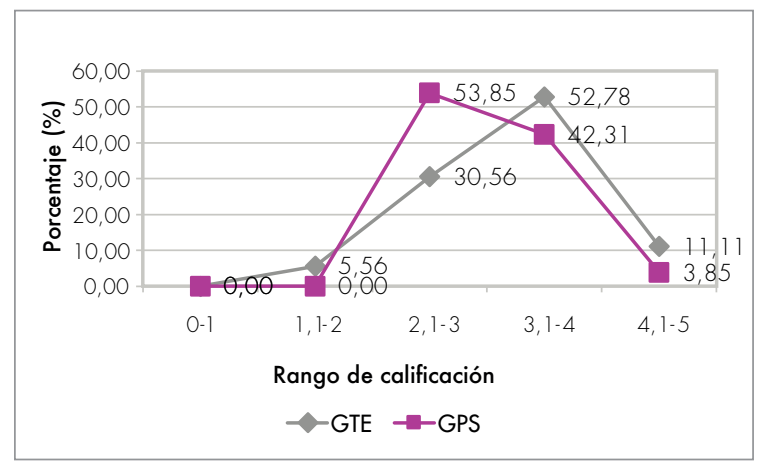

Figura 2. Relación entre el porcentaje y las calificaciones obtenidas por los dos grupos en el postest

Fuente: elaboración propia.

En la figura 2 se observa que para los dos grupos hay un aumento considerable de estudiantes agrupados en los rangos 3, 1-4 y 4, 1-5, que en los resultados del test de ideas previas no disponía de datos, pues los alumnos se incluían en los niveles inferiores. Sin embargo, para esta prueba, en el GTE el nivel de logro alcanzado es ligeramente superior que el GPS — con una diferencia de 0,3 con respecto a la media-, debido a que se evidencia un menor porcentaje de estudiantes en el rango reprobatorio 2,1-3 y se consigue un mayor incremento en las categorías superiores de aprobación. Por consiguiente, el nivel de conocimiento de la mayoría de estudiantes del GTE $(52,78 \%)$ está en la escala satisfactoria, es decir que comprenden los conceptos acerca de los factores que afectan la velocidad de las reacciones químicas, pero tienden a confundirse cuando los aplican en un problema; mientras que en el GPS, con un 53,85\% se dispone en la escala aceptable, asumiendo que estos estudiantes presentan dificultades para relacionar los conceptos en situaciones problémicas. También es importante considerar el hecho de que en el GPS no hay alumnos en los rangos inferiores, no obstante, en el grupo GTE se dispone de un porcentaje mínimo de 5,56\% (dos estudiantes) en el nivel no satisfactorio.

Análisis del pre/postest considerando la distribución de los resultados

La figura 3 presenta información acerca de la tendencia central, simetría y dispersión de las respuestas del pretest y postest de los dos grupos. 


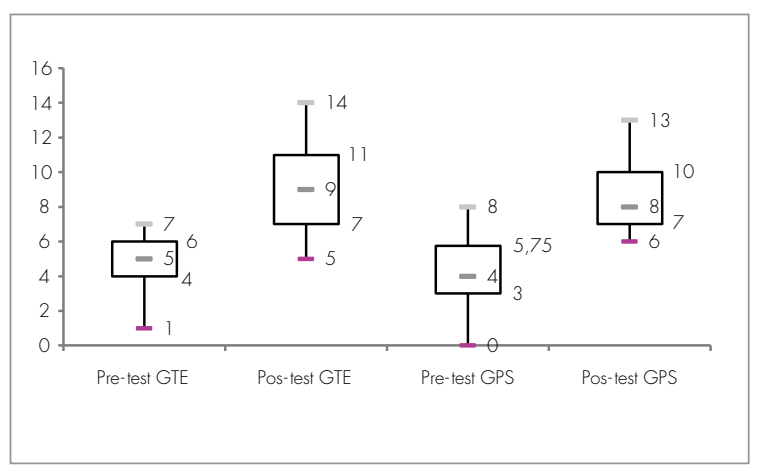

Figura 3. Distribución de resultados del pre/ postest de los dos grupos

Fuente: elaboración propia.

Los diagramas de cajas y bigotes del pretest permiten identificar en forma general dos aspectos importantes para las dos secciones que participan en esta investigación (GTE y GPS). El primer aspecto es que los grupos presentan ideas ingenuas sobre el concepto de cinética química, debido a que ningún estudiante obtuvo un resultado de más del $60 \%$ favorable, pues de catorce preguntas el máximo puntaje para el GTE fue de siete respuestas correctas y en el GPS fue de ocho; además, hay estudiantes con puntajes muy bajos, en donde solo se responde correctamente una sola pregunta como es el caso del GTE, e incluso la existencia de un puntaje de cero para un estudiante del otro grupo. Como segundo aspecto se tiene que los grupos son relativamente homogéneos debido a que el rango intercuartílico del GTE es de 2 y el de GPS es de 2,75, sin embargo, en este último grupo hay una dispersión particular en aquellos que están por encima de la media (asimetría $=-0,625$ ). Todo lo anterior trae como conclusión que la respectiva estrategia didáctica que se aplica a los dos grupos es pertinente para comparar el grado de eficiencia de cada una de ellas, ya que se parte del hecho de que son grupos similares y con ideas ingenuas sobre el concepto de cinética química; por lo tanto el postest sería un buen indicador para comparar cuál es la versión de la estrategia didáctica que mejor se adapta para el aprendizaje de dichos conceptos.

Por su parte los diagramas del postest se ubican más hacia la parte superior en relación con el pretest, esto quiere decir que los resultados obtenidos en la prueba final son significativos y, por consiguiente, la estrategia aplicada tiene un impacto positivo en el aprendizaje de los estudiantes.

\section{Fase de reflexión}

\section{Análisis general de la propuesta}

Teniendo en cuenta los resultados de las dos secciones, se evidencia que la propuesta educativa empleada en sus dos versiones metodológicas tiene una influencia positiva en la enseñanza-aprendizaje de los conceptos de cinética química. Sin embargo, es importante destacar un mayor predominio de actitudes y habilidades por parte del GTE que, sin lugar a dudas, incidieron en la leve superioridad de los resultados de la prueba final. Por consiguiente, se puede argumentar que el proceso llevado a cabo a través del aprendizaje activo basado en la utilización del trabajo práctico, le permite al maestro disponer de herramientas didácticas dinámicas, que inciden en la atención, interés y motivación de los estudiantes; factores que se lograron ver durante el desarrollo de los talleres y que en clases impartidas antes de aplicar la propuesta no se manifestaban, pues los estudiantes mantenían una actitud ajena al aprendizaje de la química, escuchando pasivamente lo que el docente decía. Es importante destacar también que a través de la observación secuencial del comportamiento del GTE, se pudo comprobar que estos estudiantes en comparación a los del otro grupo preguntaban más y no se guardaban nada; acciones que promueven el desarrollo de la investigación y la creatividad. Por su parte, en el GPS, con 
resultados favorables, el ambiente de la clase cambió con relación a sesiones anteriores, de manera que se transitó desde un contexto de poca participación hasta una atmósfera de interacción, discusión y debate. De este modo, en los dos grupos se logra introducir un escenario propicio para contrastar predicciones, crear conclusiones y el desarrollo de competencias comunicativas.

\section{Conclusiones}

Los talleres de experimentación y simulación estructuradas bajo el enfoque metodológico de aprendizaje activo, son una estrategia ideal para desarrollar en los estudiantes la adquisición de capacidades intelectuales y procedimentales, que van desde las más simples — como observar, medir, usar materiales, explorar y predecir - hasta las más complejas — confrontar ideas, debatir, analizar, sintetizar conceptos y concluir-.

La metodología del aprendizaje activo se ajusta a los principios del modelo constructivista de la enseñanza de las ciencias, en especial de la química. Por consiguiente, los estudiantes son capaces de relacionar lo que ya saben con los elementos nuevos, utilizando la ayuda del profesor que actúa como orientador, y además apoyándose en sus compañeros, debido a que en este proceso se aprende con y de los demás.

El uso de las TIC en la enseñanza de la química se constituye en un material fundamental que permite apoyar el descubrimiento y la construcción de los conceptos y habilidades a través de la interacción con herramientas virtuales de aprendizaje, como es el caso de los laboratorios virtuales. En particular, el simulador de experimentos de cinética química Virtual LabCin posibilitó el trabajo activo y dinámico de las actividades de manera personalizada, facilitó la investigación por descubrimiento, la resolución de problemas y proporcionó la forma interactiva de modelar procesos químicos reales en donde el alumno podía repetir la actividad con una inversión baja, es decir, sin la necesidad de utilizar materiales y reactivos que a veces por falta de recursos resulta difícil conseguir.

El diseño y la implementación de las actividades con aprendizaje activo acerca de la temática de cinética química, se fundamenta en la necesidad de recuperar la enseñanza de contenidos que, como este, no tienen relevancia en el plan de estudio de química en la educación media, debido a que son considerados temas difíciles que se alejan del interés de alumnos y docentes. De esta forma, la estrategia se presenta como una herramienta didáctica adecuada para la apropiación de conceptos en donde interviene la velocidad de una reacción química.

Los estudiantes traen consigo ideas alternativas erróneas acerca de los conceptos de cinética química, que debido a su abstracta comprensión, se incorporan en la estructura cognitiva de los alumnos, generando conflictos para ser conectados con la nueva información. De esta manera, se encontró que la preconcepción falsa que se tiene sobre la energía de activación no es consistente 
con las explicaciones impartidas, y a pesar de que se excluye la utilización de una estrategia tradicional, se determinó que en los dos grupos de estudio esta idea se resiste al cambio y permanece en algunos de los estudiantes.

La estrategia didáctica, en general, tiene un impacto positivo en el aprendizaje de los conceptos relacionados con los factores que afectan la velocidad de las reacciones químicas, bajo el enfoque del aprendizaje activo, debido a que no solo proporciona el desarrollo de nuevas capacidades mentales y actitudinales en los estudiantes de los grados décimos de la IESL, sino que también le permite al profesorado en química reflexionar sobre su práctica educativa, innovar su metodología y dar respuesta puntual a la situación problemática en la enseñanza-aprendizaje de los conceptos abordados.

\section{Referencias}

Acher, A. (2014). Cómo facilitar la modelización científica en el aula. Tecné, Episteme y Didaxis: TED, (36), 63-75.

Ahiakwo, M. e Isiguzo, C. (2015). Students' conceptions and misconceptions in chemical kinetics in port harcourt metropolis of Nigeria. AJCE, 5(2), 1 12-130.

Alarcón, M., Lakhdar, Z., Culaba, I., Lahmar, S., Lakshminarayanan, V., Mazzolini, A., Maquiling, J., y Niemela, J. (s. f.). Active learning in optics and photonics (ALOP): A model for teacher training and professional development. Recuperado de http://www. unesco.org/new/fileadmin/MULTIMEDIA/ HQ/SC/pdf/ALOP.pdf.

Albadalejo, M. y Caamaño, A. (1992). "Los trabajos prácticos". La resolución de problemas. En Didáctica de las ciencias de la naturaleza. Curso de actualización científica y didáctica (caps. 5 y 6, pp. 95-157). Madrid: MEC.
Barberá, O. y Valdés, P. (1996). El trabajo práctico en la enseñanza de las ciencias: una revisión. Enseñanza de las Ciencias, 14(3), 365-379.

Barrera, J. (2013). Enseñanza de los factores que afectan la velocidad de reacción: una propuesta de aula desde el aprendizaje activo, Bogotá (Colombia). Revista Horizontes Pedagógicos, 15(1), 174-180.

Bender, G., Cutrera, G. y Defago, A. (2007). Cinética química y analogías un análisis de las propuestas de enseñanza. Jornadas de Enseñanza e Investigación Educativa en el campo de las Ciencias Exactas y Naturales, $10 \mathrm{p}$.

Bigger, S. (2011). ChemKinetics: Fundamental chemical kinetics principles and analysis by computer simulation. Journal Chemical Education, 88(2), 244-244.

Bojórquez, J., López, L., Hernández, M. y Jiménez, E. (2013). Utilización del alfa de Cronbach para validar la confiabilidad de un instrumento de medición de satisfacción del estudiante en el uso del software Minitab. Cancún (México), Eleventh Laccei Latin American and Caribbean Conference for Engineering and Technology, $9 \mathrm{p}$.

Bonwell, C.C., y Eison, J. A. (1991). Active Learning: Creating Excitement in the Classroom (Asheeric Higher Education Report 1). George Washington University, Washington, DC.

Botero, J. y Palomeque, L. (2014). El ova como estrategia para la enseñanza aprendizaje de la cinética química. Laclo, pp. 737-740. Conferencia Latinoamericana de Objetos y Tecnología de Aprendizaje Laclo 2014.

Cachapuz, F. y Maskill, R. (1987). Detecting changes with learning in the organization of knowledge: Use of word association tests to follow the learning of collision theory. International Journal of Science Education, 9(4), 491-504. 
Cardellini, L. (2012). Chemistry: Why the Subject is Difficult? Educación en Química, 23, suppl. 2, 6 p.

Cataldi, Z., Donnamaría, C. y Lage, F. (2008). Simuladores y laboratorios químicos virtuales: Educación para la acción en ambientes protegidos. Quaderns Digitals $55,10 \mathrm{p}$.

Contreras, G. y Carreño P. (2012). Simuladores en el ámbito educativo: un recurso didáctico para la enseñanza. Ingenium, Revista de la Facultad de Ingeniería, 25, 107-119.

Cuadrado, J. (2010). Los docentes en la actualidad. Revista Digital de Innovación y Experiencias Educativas, 28(122).

Da Silva, J., Sousa, M., Silva, E., Oliveira, F. y Melo, A. (2014). KinChem: A Computational Resource for Teaching and Learning Chemical Kinetics. Journal of Chemical Education, 91 (12), 2203-2205.

Escamilla, J. (2000). Selección y uso de tecnología educativa. México: Trillas.

Golombek, D. (2008). Aprender y enseñar ciencias: del laboratorio al aula y viceversa, Buenos Aires: Fundación Santillana.

Gonzales, H. (2000). La evaluación de los estudiantes en un proceso de aprendizaje activo de la cartilla docente. Cali: Publicaciones del Crea.

Hernández, J. (2013). Implementación de las tic en la enseñanza de la cinética y equilibrio químico en los estudiantes del grado 11 de la institución educativa Emiliano García (Tesis de maestría). Universidad Nacional de Colombia, Medellín (Colombia).

Hernández, R., Fernández, C. y Baptista, P. (2003). Metodología de la investigación. Diseños experimentales. Ciudad de México: McGrawll-Hill.

Hiler, W. y Paul, R. (2006). The miniature guide on active and cooperative learning. Ideas prácticas para promover el aprendizaje activo y cooperativo: 27 maneras prácticas para mejorar la instrucción. Basado en los conceptos y principios del pensamiento crítico. Dillon Beach: Fundación para el Pensamiento Crítico. Recuperado de http://www.criticalthinking.org/resources/PDF/SP-Active_and_ coop_learning.pdf.

Huddle, P. y White, M. (2000). Simulations for teaching chemical equilibrium. Journal of Chemical Education, 77(7), 920-926.

Kaya, E. y Geban, Ö. (2012). Facilitating conceptual change in rate of reaction concepts using conceptual change oriented instruction. Education \& Science/ Egitim ve Bilim, 37, Issue 163, 216-225.

Kolomuç, A. y Çalik, M. (2012). A comparison of chemistry teachers' and grade 11 students' alternative conceptions of 'rate of reaction'. Journal of Baltic Science Education, 17 (4), 333-346. 
Kolomuç, A. y Tekin, S. (2011). Chemistry Teachers' Misconceptions Concerning Concept of Chemical Reaction Rate. Eurasian Journal Physics Chemistry Education, 3(2), 84-101.

Kurt, S. y Ayas, A. (2012). Improving students' understanding and explaining real life problems on concepts of reaction rate by using a four step constructivist approach. Energy Education Science and Technology Part B: Social and Educational Studies, 4(2), 979-992.

Lynch, M. D. (1997). The effect of cognitive style, method of instruction, and visual ability on learning chemical kinetics (Tesis Ph. D. no publicada). lowa State University, Estados Unidos.

Martínez, L. (2009). Enseñanza constructivista sobre conceptos de cinética en la formación inicial de profesores de Química. Educación Química, Formación de Profesores, 20 (3), 383-392.

Ministerio de Educación Nacional-Colombia. (2004). Formar en ciencias: iel desafío! Serie guías, $7.48 \mathrm{p}$.

Narváez, L. (2015). Propuesta para la enseñanza-aprendizaje de balanceo de ecuaciones químicas implementando simuladores para estudiantes de grado décimo de la Institución Educativa Samaria. (Tesis de maestría). Universidad Nacional de Colombia, Manizales (Colombia).

Oviedo, H. y Campo, A. (2005). Aproximación al uso del coeficiente alfa de Cronbach. Revista Colombiana de Psiquiatría, XxxIv(4), 572-580.

Prince, M. (2004). Does active learning work? A review of the research. Journal of Engineering Education, 93(3), 223-231.
Ramos, D. (2013). Recursos y estrategias para un aprendizaje activo del alumno en el aula de ElE. Actas del I Congreso Internacional de Didáctica del Español como Lengua Extranjera, Instituto Cervantes de Budapest, 405 p.

Rodríguez, J., Mayén, M., Rodríguez, R. y Ruiz, M. (2013). Preparing the students for the laboratory by means of virtual labs. Journal of Laboratory Chemical Education, $7(2), 19-24$.

Sánchez, J., Domínguez, J. y García, E. (2002). Revisión de la investigación sobre la enseñanza de la cinética química. Adaxe-Revista de Estudios y Experiencias Educativas, 18, $171-190$.

Sierra, H. (2013). El aprendizaje activo como mejora de las actitudes de los estudiantes hacia el aprendizaje (Trabajo final de máster). Universidad Pública de Navarra.

Sokoloff, D. (2006). Active learning in optics and photonics: training manual. United Nations Educational, Scientific, and Cultural Organization-Unesco; International Centre for Theoretical Physics-ICTP; The International Society for Optical Engineering-SPIE.

Stieff, M. y Wilensky, U. (2003). Connected chemistry incorporating interactive. Simulations into the chemistry classroom. Journal of Science Education and Technology, 12(3), 285-302.

Tastan, Ö., Yalçinkaya, E. y Boz, Y. (2010). Pre-service chemistry teachers' ideas about reaction mechanism. Journal of Turkish Science Education, 7(1), 47-60.

Tejada, C., Chicangana, C. y Villabona, A. (2013). Enseñanza de la química basada en la formación por etapas de acciones mentales (caso enseñanza del concepto de valencia). Revista Virtual Universidad Católica del Norte, 38, 143-157. 
Useda, P. L. y Castañeda, M. A. G. (2015). El ambiente digital en la comunicación, la actitud y las estrategias pedagógicas utilizadas por docentes. Tecné, Episteme y Didaxis, 37, 109-129.

Wu, H. (2003). Linking the microscopic view of chemistry to real life experiences: intertextuality in a high school science classroom. Science Education, 87, 868-891.

Zain, S., Abdul, N. y Sui, L. (2013). The teaching and learning of chemical kinetics supported with MS Excel. Journal of Science and Mathematics Education in Southeast Asia, 36(1), 65-85.

\section{Para citar este artículo}

Paredes, J. y Molina, M. (2019). Enseñanza de la cinética química por medio de simulaciones y aprendizaje activo. Tecné, Episteme y Didaxis: TED, 45, 71-88. 